\title{
Measuring Color Perception through Laser Mitigation Coatings on Aircraft Windshields
}

\author{
Stanley L. Harriman, Randal J. DeMik, Ryan S. Phillips, Suling Li, Megan M. \\ Zahos, Nick P. Kazanjian, Ehteshamuddin Arif, Mark D. Wolfrum, Joseph E. \\ Burlas, and Bobby Hill, Jr. \\ Lewis University
}

\begin{abstract}
Ground-based laser illuminations directed towards arriving and departing aircraft have increased in the past decade. A laser aimed at the windshield of an aircraft may distract a pilot and compromise safety. Previous studies provided empirical evidence of laser intensity in the flight deck from ground-based laser illumination events and the potential for adverse effects to flight safety. Most recent studies focused on testing the application of various coatings to aircraft windshields in order to reduce the effects of laser exposure to crewmembers. Safe and efficient flight operations depend on the ability of a pilot to see normal spectrums of color. Therefore, this study used the Ishihara Pseudoisochromatic Plates Color Vision Test to investigate participants' color perception through an aircraft windshield coated with a photoresponsive nanocomposite film designed to reduce laser intensity from entering a flight deck. This study tested the hypothesis that there were no differences between color vision test scores when conducting trials with coated and noncoated windshields. Participants were individuals who held a current FAA medical certificate and held a minimum of a student pilot certificate $(N=104)$. Data analysis consisted of a repeated measures design that included within-subjects factors where each of the participants was tested from two trials, each under two conditions: coated and noncoated. The order of trials was altered using a counterbalancing technique which also provided a between-subjects factor. A paired-samples $t$-test was calculated to compare the mean of error by participants when taking the Ishihara Test through the non-coated windshield to the mean of the error by participants when taking the test through the coated windshield. No significant difference from the non-coated to the coated windshield was found $(t(103)=1.274, \mathrm{p}>0.05, \mathrm{n}=104)$. Findings suggest that effective color vision can be maintained through photoresponsive nanocomposite coatings.
\end{abstract}

\section{Introduction}

The safety record of aviation continues to improve with the number of fatal airline accidents in 2011 being the lowest in the last decade (Ranter, 2011). However, over the past several years laser illuminations directed at aircraft have increased in frequency and have led to safety concerns for the aviation community (Leiser, 2011). According to the Federal Aviation Administration's (FAA) Laser Safety Initiatives (2013), reports indicated a nine-fold increase in laser incidents between the years 2006 and 2012. For this study, laser illumination was defined as both the intentional and unintentional act of a laser beam striking an aircraft windshield during any phase of flight or ground operation. In a study by DeMik et al (2013) researchers utilized lasers which are readily available to the public, and shone them through three untreated aviation windshields. Researchers discovered that 
the highest potential risk to flight safety during illumination events is not necessarily eye injury, but the possibility of flight crews becoming distracted during critical phases of flight below 2000 feet.

Advancements in technology have been investigated in recent years in an attempt to reduce the potential dangers associated with laser illumination events that included the use of protective eyewear, smart goggles, and glare shields (Murphy, 2009). There is a patent pending on an active Laser Protection System (LPS) and was developed to provide an automatic adjustment response to laser light exposure and was developed in the form of both a visor and contact lens (Harvie, 2007). Lastly, glare shields can be used and deployed in the event of laser illumination events to reduce exposure.

One possible mitigation tactic is the use of protective eyewear on the flight deck. The benefits of eyewear may be outweighed by the consequences of the operational restrictions imposed on pilots. Laser eye protection in the form of filtered spectacles, visors and goggles can alter the environment in which pilots operate (Svec, 2005). Protective eyewear must be capable of allowing a normal spectrum of color and quantity of light to the pilot's eyes while blocking only the specific wavelengths associated with laser illumination events (Stewart, 2005). This may be challenging considering the availability of lasers in numerous hues and the necessity of ensuring there is no negative impact on color vision as colors associated with multi-function displays, electronic flight displays and annunciators are vital to the safe operation of the aircraft. "One case involved aircrew wearing yellow visors attempting to land on an unusable portion of the runway, they were unable to see the large yellow ' $\mathrm{X}$ ' indicating the hazard" (Svec, 2005, p. 42).

A pilot's ability to discern color is needed for safe operation of an aircraft in critical phases of flight. Perception of color is necessary during the takeoff and landing phases of flight to differentiate and recognize items such as aircraft position lights, airport beacons, runway lights, approach slope indicators, light gun signals and obstruction lighting. Additionally, Part 67 of the Federal Aviation Regulations sets medical standards and certification requirements for airmen and mandates pilots to possess the ability to perceive those colors necessary for the safe performance of airman duties for all classes of medicals (FAA, 2015).

In a study conducted using active duty U.S. Air Force airmen, seven volunteers underwent comprehensive color vision testing to research the impact of Short Wavelength Absorbing Filters (SWAFs), such as High Contrast Visors (HCV), and selected waveband type Laser Eye Protection (LEP) devices on color vision (Young et al, 2000). These devices proved to significantly degrade color vision. Further research on low and bright illuminant conditions and the resulting impact on color vision were recommended.

Additional research in the medical and nursing field has demonstrated similar effects. One study indicated that laser protection devices may distort color perception by blocking the transmittance of specific light wavelengths (Teichman et al, 1999). Researchers tested the color confusion of urologists when wearing laser eye protection for laser soft tissue 
applications. They found that laser eye protection devices for potassium titanyl phosphate caused significant blue-yellow and red-green confusion.

It is noteworthy to mention that the use of visors, glasses, and glare shields are considered to be active mitigation countermeasures. Pilots must actively utilize these devices to protect themselves from laser illuminations. This may prove problematic during critical phases of flight, as it adds one additional item to a task-saturated environment. Considering this issue, Phillips et al (2015) recommended the use of a passive system, requiring no additional action from the pilots, through permanent photoresponsive nanocomposite films that are applied directly to an aircraft windshield to reduce the visual effects of laser illuminations. The coating selected for this current study was identified by researchers as having the most meaningful reduction in laser intensity with a mitigation effectiveness of approximately 80 percent (Phillips et al, 2015).

\section{Statement of the Problem}

The Federal Bureau of Investigations disclosed 3,960 FAA-reported laser illuminations directed towards aircraft in 2013, an increase from 384 reported incidents in 2006 (FBI, 2014). In response to the increase in laser illuminations over the previous decade, President Obama signed into public law the FAA Modernization and Reform Act of 2012, deeming it a federal crime to aim a laser at an aircraft. Despite these measures, laser illuminations continue to be a safety concern for aviation flight crews (FAA, 2013). At the time of this study, no previous research has been conducted to determine the effects of passive means, such as protective coatings on windshields, as related to color perception. In response to this problem, researchers investigated the impact of photoresponsive nanocomposite windshield coatings on participants' color perception.

Researchers in the study measured the impact of a coated windshield versus a noncoated windshield on the participant's color perception in an effort to answer the following hypotheses were tested:

1. There is no difference in the number of errors utilizing the Ishihara Color Test when conducting trials as viewed through coated and non-coated windshields.

2. There is no difference in the amount of time to complete the Ishihara Color Test when conducting trials as viewed through coated and non-coated windshields.

3. There is no difference in the number of errors utilizing the Ishihara Color Test when conducting trials based on the order of the windshield coating (non-coated vs. coated). 


\section{Methodology}

\section{Sample Population}

Participants included only individuals who possessed a current FAA medical certificate with no restrictions for color vision and a minimum of a student pilot certificate. A total of 104 participants consisting of Lewis University students and instructors were tested. Participants ranged in age from $18-45$ years old. Seven percent of participants in this study identified themselves as female and 93 percent of participants identified themselves as male.

\section{Instrumentation}

This study used Boeing 737 windshields, one coated with a laser protecting film and one non-coated windshield. The film selected was the most effective photoresponsive nanocomposite coating developed out of three films tested in a 2015 study by Phillips et al.. The Ishihara Test used by FAA medical examiners to test pilots for color perception was applied to conduct trials and calculate color vision performance scores. According to a validation study done by the FAA in 1993, the Ishihara Pseudoisochromatic 24-plate Color Vision Test has a respectable reliability with an alpha value of 0.98 . Test-retest reliability was found to be $\mathrm{k}=1.00$, therefore the FAA has accepted the Ishihara Test to provide consistent performance in aeromedical screening (FAA, 1993).

\section{Data Collection}

Participation in this study was strictly voluntary in nature and recruitment was in the form of classroom announcements, emails, and informational posters displayed at Lewis University. Participants were presented with a letter of consent and debriefed after each trial to remind them that participation and inclusion of their data was voluntary as approved by the Lewis University Institutional Review Board (IRB).

Each trial consisted of two tests: a test utilizing the non-coated windshield condition (control); and another under the coated windshield condition. The order of taking the tests was alternated among participants as a counter-balancing technique. The time required for each individual to take each test in its entirety was recorded in an attempt to determine if time is a significant factor in the success of each test. Participants identified the object or number designated on each color plate viewed through each windshield (Fig. 1). A researcher recorded both performance and time required while testing each windshield during the trial. In order to maintain the integrity of the Ishihara Test, registered nurses $(\mathrm{RN})$, who were familiar with colorblindness testing procedures, conducted trials for each participant. 


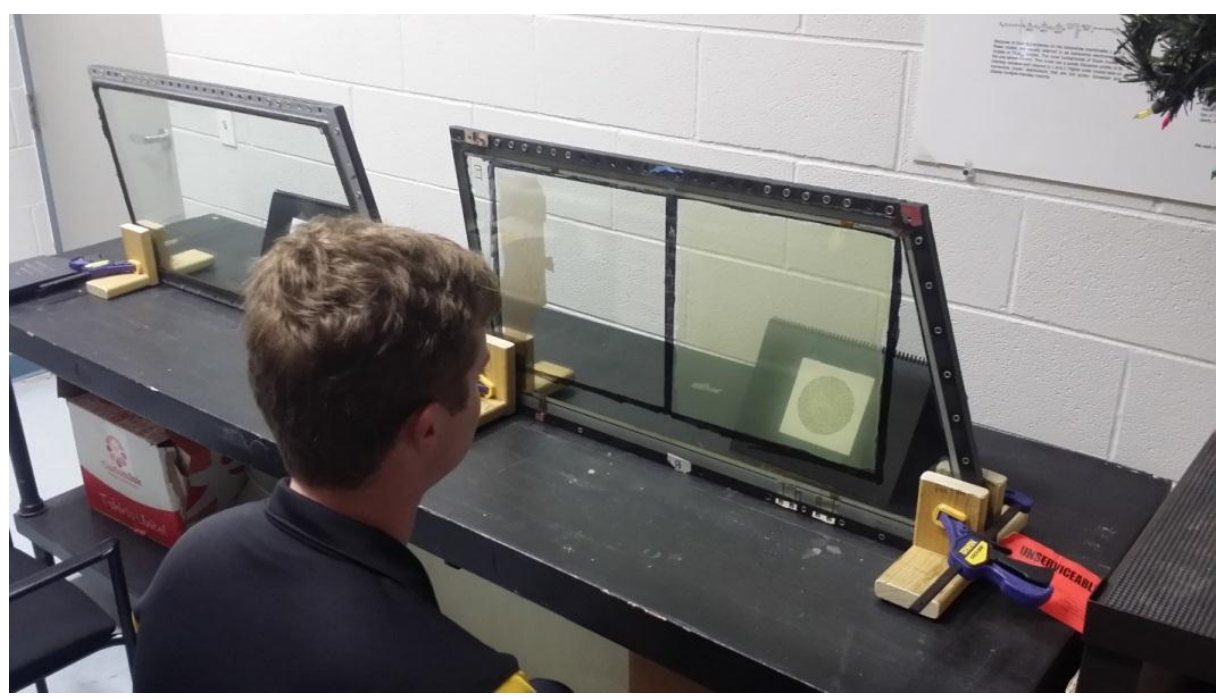

Figure 1. Photo representative of testing apparatus and participant.

Researchers utilized the Ishihara Test and testing was conducted according to standards set forth by the FAA (Fig. 2). The FAA has outlined the following techniques for all medical examiners (FAA 2014):

1. Test book should be held 30 inches from the participant;

2. Plates should be illuminated by at least 20 candles (or equivalent), preferably by a Macbeth Easel Lamp or a Verilux True Color Light;

3. Only three seconds are allowed for the participant to interpret and respond to a given plate; and

4. Participants are only tested using plates 1 to 15 .

Researchers recorded and calculated responses given by each participant as a score ranging from 0 to 15 (correct response for each plate signifying one point) in order to determine any differences in color perception between the two trials using coated and uncoated windshields. 


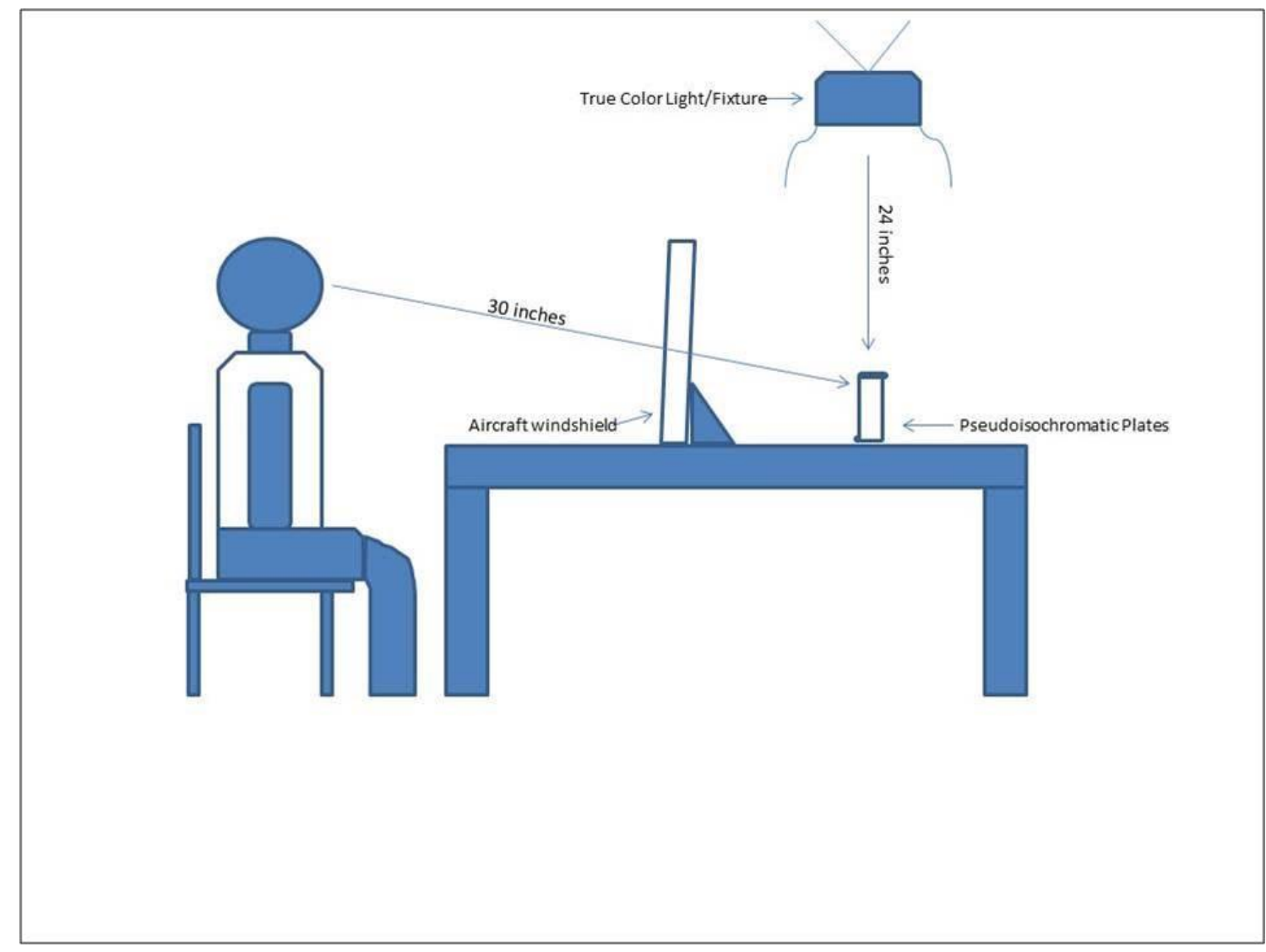

Figure 2. Depiction of participant and testing equipment during trials.

\section{Statistical Analysis}

The characteristics of this research study were consistent with a repeated measures design that included within-subjects factors where each of the participants provided data from two trials, each under a different condition (coated or non-coated). The order of trials (coated first or non-coated first) was altered using a counterbalancing technique which provided a between-subjects factor. All data for this study was entered into SPSS for analysis. The dependent variables were analyzed using a paired-samples $t$ test to provide initial analysis for this study. There was also a between-subjects factor of order of trial.

The Type I error for this investigation was set at $0.05(\alpha=0.05)$. Minium, Clarke, and Coladarci (1999) noted that the Type I error of 0.05 is the most commonly used for this type of research. While this alpha gives a higher probability of incorrectly accepting a false alternative hypothesis (Type I error) than an alpha of 0.01 , the results of this study were informational only. In this case the risk involved in a Type I error was small, and reducing the Type I error to 0.01 was not necessary. 


\section{Results}

The findings of the data analysis support the stated hypothesis on each count.

\section{Error}

There was no difference in the number of errors utilizing the Ishihara Color Test when conducting trials as viewed through coated and non-coated windshields. A paired-samples $t$ test was calculated to compare the mean of error by participants when taking the Ishihara Test through the non-coated windshield to the mean of the error by participants when taking the test through the coated windshield. The mean on the non-coated windshield was $0.24(s d=0.55)$ and the mean on the coated windshield was $0.34(s d=0.65)$. No significant difference from the non-coated to the coated windshield was found $(t(103)=1.274, \mathrm{p}>$ $0.05, \mathrm{n}=104)$.

\section{Time}

There was no difference in the amount of time to complete the Ishihara Color Test when conducting trials as viewed through coated and non-coated windshields. A pairedsamples $t$ test was calculated to compare the mean of time utilized by participants when taking the Ishihara Test through the non-coated windshield to the mean of the time utilized by participants when taking the test through the coated windshield. The mean on the noncoated windshield was 25.03 seconds $(s d=4.82)$ and the mean on the coated windshield was 25.45 seconds $(s d=4.34)$. No significant difference from the non-coated to the coated windshield was found $(t(103)=0.929, \mathrm{p}>0.05, \mathrm{n}=104)$.

\section{Order}

There was no difference in the number of errors utilizing the Ishihara Color Test when conducting trials based on the order of the windshield coating (non-coated vs. coated).

An independent samples $t$ test was calculated comparing the mean score of participants who took the Ishihara Test through the coated windshield first to the mean score of participants who took the test through the non-coated windshield first. No significant difference was found $(\mathrm{t}(102)=-2.795, \mathrm{p}>0.05, \mathrm{n}=104)$. The mean of the coated windshield time first $(\mathrm{m}=24.32$, $\mathrm{sd}=3.81)$ was not significantly different from the mean of the non-coated windshield time first $(\mathrm{m}=23.73, \mathrm{sd}=4.58)$. Of the 104 participants, 53 were tested with the coated windshield first and 51 with the non-coated windshield first.

\section{Discussion of the Results}

The purpose of this study was to provide empirical evidence that the photoresponsive nanocomposite windshield coating does not negatively affect a participant's color perception. Researchers' specific goal was to evaluate if participants would demonstrate differences between color perception test scores when conducting trials utilizing coated vs

non-coated windshields as a factor of: 1) overall errors made; 2) time recorded while taking 
each test; and 3) order of trials between windshields tested. Researchers hypothesized that the coating would demonstrate no significant impact on color perception errors on the Ishihara Test. Findings suggest that effective color vision can be maintained through photoresponsive nanocomposite coatings.

There is limited research regarding the impact of laser protective coating applications to windshields regarding the color perception of a user; however supporting research on protective eyewear or visors has demonstrated degradations to color perception (Young et al, 2000; Svec, 2005). Results from this study provide empirical evidence that the coating tested does not significantly affect participants' color perception. Although the windshield

coating tested supported researchers' hypotheses, participants in the study did however provide comments that indicate further research into a more effective coating is necessary. Remarks included that the coated windshield was "blurry and tinted, but colors [were] unaffected" and that the coating "doesn't distort color but makes viewing hazy." Continued increases in reported intentional laser illuminations over the last decade lends further support to additional research on the development of an effective coating that does not distort perception.

The study limitations merit comment, specifically the environmental limitations. Trials for this study were administered within a controlled environment, established by the FAA as outlined in the Guide for Aviation Medical Examiners (FAA, 2014). Provisions for this study do not simulate all types of environmental conditions (weather, time of day, changes in ambient light) or other external stressors (fatigue or stress) which may exist during various phases of flight. Future research designs may benefit from the inclusion of varying weather and lighting conditions to better understand the coating's impact on color perception.

\section{Recommendations for Future Research}

The evidence available on the impact of laser illumination events on aircraft demonstrates that further research into the subject matter is both valuable and necessary. While this study suggests that the tested windshield coating does not significantly affect color perception, further research is required to demonstrate that the coating does not negatively impact the overall visibility and light available in the flight deck. Comments by participants regarding the blurriness of the film warrant investigation into various application techniques to improve clarity. In addition to investigating the windshield's coating on overall dilution or filtration, further exploration may be necessary regarding the effect that environmental conditions may have on life of the coating. Thermal testing would provide valuable information on the efficacy of the practical application of research in the commercial aviation environment. 


\section{Acknowledgements}

Special thanks to Dr. Jason Keleher and his research team at Lewis University for providing the photoresponsive nanocomposite coating used in this study. This research was sponsored, in part, by a grant from The Colonel Stephen S. And Lyla Doherty Center for Aviation and Health Research. 


\section{References}

DeMik, R., Harriman, S., Phillips, R., Crowder, C., Pfeiffer, J., De Waard, E., McHugh, S., Foster, S., Streit, M., Antoniolli, R., Knight, E., York, D., \& Luedtke, J. (2013). Measuring intensity of laser light penetrating flight decks in laser illuminations. Journal of Aviation Technology and Engineering, 3(1). 1-8. DOI: 10.7771/21596670.1068

Federal Aviation Administration. (1993). Validity of FAA-approved color vision tests for class II and class III aeromedical screening. Springfield, Virginia: Federal Aviation Administration.

Federal Aviation Administration. (2013). Laser Safety Initiative. Retrieved from http://www.faa.gov/about/initiatives/lasers/

Federal Aviation Administration. (2014). Guide for medical examiners. Retrieved from http://www.faa.gov/about/office_org/headquarters_offices/avs/offices/aam/ame/guide /media/guide.pdf

Federal Aviation Administration. (2015). Federal Aviation Regulations Aeronautical Information Manual. Newcastle, Washington: Aviation Supplies and Academics.

Federal Bureau of Investigation. (2014). FBI pilots new reward program aimed at deterring laser strikes. Retrieved from http://www.fbi.gov/news/pressrel/pressreleases/fbi-pilots-new-reward-program-aimed-at-deterring-laser-strikes

Harvie, M.R. (2007). Active laser protection system, U.S. Patent 7202852.

Leiser, K. (2011). Incidents of lasers vs. aircraft hit a record. Retrieved from http://www.stltoday.com/news/local/metro/incidents-of-lasers-vs-aircraft-hit-arecord/article_3aa8aadc-df4c-5e0b-907e-efd1ce6e0936.html

Minium, E. W., Clarke, R.C., \& Coladarci, T. (1999). Element of statistical reasoning $\left(2^{\text {nd }}\right.$ ed.). New York, New York: John Wiley.

Murphy, P. (2009, September 10). Lasers and Aviation Safety. International Laser Display Association. Retrieved from http://www.laserist.org/files/Lasers-andaviation-safety_2pt2.pdf

Phillips, R.S., Bilan, H.K., Widel, Z.X., DeMik, R.J., Brain, S.J., Moy, M., Crowder, C., Harriman, S.L., O’Malley III, J.T., Burlas, J.E., Emmert, S.F., \& Keleher, J.J. (2015). Measuring the effectiveness of photoresponsive nanocomposite coatings on aircraft windshields to mitigate laser intensity. Journal of Aviation Technology and Engineering, 4(2), 49-54. DOI: 10.7771/2159-6670.1105 
Ranter, H. (2011). ASN releases preliminary safety statistics 2011. Flight Safety Information. Retrieved from http://aviation-safety.net/news/newsitem.php?id=2481

Stewart, C. (2005). Weapons of mass casualties and terrorism response handbook. Retrieved from http://books.google.com/books?id=7ZnXZfwWwgcC\&pg=PA220\&lpg=PA220\&dq= laser+protective+goggles+pilots\&source $=$ web\&ots=Nzq4sWnWNw\&sig=sem-g858T k7KyOUddmEQUqXrPY\&hl=en\&sa=X\&oi=book_result\&resnum=10\&ct=result\#v= onpage\&q=laser\%20protective\%20goggles\%20pilots\&f

Svec, L. (2005). The effect of spectrally selective filters on color perception and aviation performance. Doctoral dissertation. Retrieved from ProQuest Digital Dissertations. (AAT 305429655)

Teichman, J. M. H., Vassar, G. J., Yates, J. T., Angle, B. N., Johnson, A. J., Dirks, M. S., \& Thompson, I. M. (1999). Color vision deficits and laser eyewear protection for soft tissue laser applications. The Journal of Urology, 161(3), 874-880.

Young. P.A., Perez-Becerra, J., \& Ivan, D. (2000). Aircrew visors and color vision performance: a comparative and preliminary pilot study analysis. Aviation Space and Environmental Medicine, 71(11), 1081-1092. 